Instituto Internacional de Investigación y Desarrollo Tecnológico Educativo INDTEC, C.A.

DOI: https://doi.org/10.29394/scientific.issn.2542-2987.2016.1.2.6.89-109

OAI-PMH: http://www.indteca.com/ojs/index.php/Revista Scientific/oai

\title{
Aproximación Teórica al Uso del Proyecto Educativo Canaima como Herramienta para Fortalecer la Calidad Educativa
}

\author{
Autora: Eglis Coromoto Quintero Gómez \\ Universidad Nacional Experimental "Rafael María Baralt", UNERMB \\ eglis2311@hotmail.com \\ Zulia, Venezuela
}

\section{Resumen}

La investigación, tiene como propósito generar una aproximación teórica que fundamente el uso del Proyecto Educativo Canaima como herramienta para fortalecer la calidad educativa en las escuelas primarias ubicadas en la parroquia Marcelino Briceño, municipio Baralt, estado Zulia. La misma se realizará en las escuelas primarias ubicadas en la parroquia Marcelino Briceño, municipio Baralt. Las teorías referenciales se sustentarán en González (2012), Centeno y otros (2011), entre otros. Para su desarrollo se asumirá el enfoque cualitativo a través del método fenomenológico sustentado en la hermenéutica que permitirá la comprensión de los fenómenos en sus variadas manifestaciones. Los informantes claves se seleccionarán siguiendo criterios propios de la investigación cualitativa, partiendo del hecho que son docentes de las escuelas antes mencionadas y se comprometieron a participar voluntariamente en la investigación. De tal manera se considerarán como informantes cinco docentes de aula. Para el desarrollo del estudio se utilizarán como instrumentos una entrevista no estructurada, grabaciones de audio, que permitirán estudiar en profundidad las unidades de análisis. Los hallazgos permitirán generar la construcción teórica antes mencionada.

Palabras clave: proyecto educativo Canaima; calidad educativa; educación primaria. 


\title{
Theoretical Approach to the Use of Educational Project as a Tool to
} Strengthen Canaima Education Quality

\begin{abstract}
The research aims to generate a theoretical approach that substantiates the use of Canaima Education Project as a tool to strengthen the quality of education in primary schools in the parish Marcelino Briceño, municipality Baralt, Zulia state. It will take place in primary schools in the parish Marcelino Briceño, municipality Baralt. Referential theories will be based on González (2012), Centeno et al (2011), among others. For the qualitative development through the phenomenological method supported by hermeneutics that enable understanding of the phenomena in its various manifestations approach is assumed. Key informants will be selected according to criteria of qualitative research, based on the fact that they are teachers of the aforementioned schools and pledged to voluntarily participate in the research. So they shall be considered as informants five classroom teachers. For the development of the study will be used as instruments an unstructured interview, audio recordings, that will study in depth analysis units. The findings will generate the above theoretical construct.
\end{abstract}

Keywords: educational project canaima; quality education; primary education.

Date Received: 12-08-2016

Date Acceptance: 30-08-2016 


\section{Introducción}

El impacto que las TIC han tenido en la transformación de la realidad social permea los ámbitos de la vida familiar, educativa, laboral, comunitaria, política y económica. En ese sentido, la educación primaria no solamente tiene que adaptar las nuevas tecnologías a los procesos de aprendizaje, sino también ofrecer a los estudiantes los conocimientos necesarios para su adecuada utilización dentro y fuera de las aulas.

En ese marco de ideas, es indispensable ofrecer al estudiante los saberes indispensables para utilizar apropiadamente las redes sociales, los softwares educativos, la Internet, así como los entornos virtuales, para apoyar sus actividades de aprendizaje, es decir poseer las competencias tecnológicas necesarias para su uso, por cuanto estas herramientas propician el alcance de un óptimo desempeño con su adecuada aplicación.

Por ello, en esta etapa de transición de estudiante receptor de información a constructor de conocimientos, los educandos deben atender a un reto fundamental: introducir las tecnologías de información y la comunicación (TIC) en la construcción de sus aprendizajes, adquiriendo las habilidades tecnológicas necesarias para acceder no sólo al caudal de conocimientos que éstas trasmiten. Dentro de ese contexto, surge el Proyecto Educativo Canaima, el cual nace de los compromisos del Estado venezolano para dar respuesta a los derechos de los/as venezolanos/as, incorporando el conocimiento, aplicación de las nuevas tecnologías a los procesos educativos como un medio para reducir la brecha social, tecnológica en el menor tiempo y costo posible, siendo por tanto una herramienta tecnológica para promover la construcción de significados contextualizados que preparen al educando a aprender de por vida, en cada escenario, gracias a su uso, dentro del aula y/o fuera de ella.

De allí que en la investigación se propone generar una aproximación teórica que fundamente el uso del Proyecto Educativo Canaima como 
herramienta para fortalecer la calidad educativa en las escuelas primarias ubicadas en la parroquia Marcelino Briceño, municipio Baralt, estado Zulia, por cuanto éste posibilita el uso de las tecnologías de información y comunicación como medio didáctico para facilitar el aprendizaje de diversos contenidos curriculares. Para ello se enmarcó en una metodología cualitativa con enfoque fenomenológico sustentándose en el análisis y la interpretación de los testimonios aportados por los informantes claves, a los cuales se sumó un proceso de reflexión, estar enmarcado en la creación de categorías emergentes que permitieron el basamento de aportes teóricos, dentro de la investigación. Las investigaciones previas relacionadas con el fenómeno de estudio se ubicaron dentro del análisis de la situación a investigar con el objeto de dar sustentación a las percepciones previas de la investigadora, indicando con ello la preexistencia de esta realidad no sólo en el ámbito de la parroquia Marcelino Briceño, sino a nivel nacional.

\section{Teoría y Conceptos}

\subsection{Aproximación al objeto de estudio}

En Venezuela se conciben las tecnologías de información y comunicación (TIC) como la representación de la tecnología tradicional constituida principalmente por la radio, televisión, telefonía convencional, al igual que la red de redes: Internet. De esa manera, se vienen implementando paulatinamente programas a nivel educativo para aumentar la capacidad de los estudiantes, buscando mejorar, adaptar los procesos de aprendizaje al auge tecnológico.

Desde esa perspectiva, durante los últimos años se ejecutaron algunos programas educativos tecnológicos, con el fin de otorgar a los estudiantes el acceso inmediato a nuevas fuentes de información, como el Plan de Desarrollo de los Infocentros venezolanos, el cual mediante decreto, se inicia en el año 2001 para el acceso a Internet, con el fin de facilitar el uso de estas 
tecnologías, fomentar el desarrollo, a partir de su integración en el sector escolar. Por esa razón, el Estado venezolano realizó grandes esfuerzos por hacer que toda la población tenga acceso a la tecnología mediante el uso pedagógico de las TIC, a través de los Centros Bolivarianos de Informática y Telemática (CBIT), los Centros de Gestión Parroquial (CGP), las Unidades Móviles para la Educación, las Superaulas, los Infocentros, entre otros.

En ese sentido, a partir del 2009 nace el Proyecto Educativo Canaima Educativo, el cual fue elaborado por el Ministerio del Poder Popular para la Educación, a través de la Dirección General de Tecnología de la Información y Comunicación para el Desarrollo Educativo (DGTICDE) con la finalidad de incorporar las tecnologías de información libre en las instituciones públicas, potenciar la enseñanza, aprendizaje de los jóvenes, incorporar las computadoras portátiles como un recurso didáctico, promover, fortalecer el aprendizaje integral, desarrollar el pensamiento crítico, reflexivo, creativo en los estudiantes.

Ahora bien, este proyecto se desarrolló con el fin de garantizar un primer contacto de los/as niños/as en edad escolar con las nuevas tecnologías. Con él se buscó equipar todas las escuelas públicas del país de computadoras portátiles con software educativo para que los estudiantes se familiarizaran con dichas tecnologías, al incluir las minilaptops en las aulas. Según Solarte (2010) este proyecto educativo busca promover la formación integral de los/as niños/as venezolanos/as; desarrollar sus potencialidades con la tecnología; romper con la metodología de enseñanza tradicional; impulsar el pensamiento crítico, creativo y reflexivo; fortalecer la pertinencia geohistórico-cultural, trabajando con el principio de integralidad en las asignaturas; así como adecuarlas en un mismo contenido.

Para la investigadora, Canaima Educativo ofrece muchas ventajas más allá de las pedagógicas, entre ellas resalta la capacidad de enseñar a los/as 
niños/as, desde temprana edad, a utilizar las tecnologías de información libres como instrumente de autoaprendizaje y desarrollo.

Para González (2012) los docentes consideran a la Canaima educativa como una herramienta tecnológica para desarrollar el proceso de enseñanza para los aprendizajes, su uso diario permite que los/as estudiantes puedan innovar, crear, despertar interés, inquietudes y difundir lo aprendido mediante la portátil. En ese sentido, pude evidenciar en visitas reiteradas a las aulas de las escuelas primarias ubicadas en la parroquia Marcelino Briceño, municipio Baralt que los/as docentes no están suficientemente preparados tecnológicamente para el uso del software libre, lo cual genera desinterés para utilizar la herramienta en clases, al consultar acerca de esa debilidad indicaron falta de preparación para aplicar y trabajar con esta herramienta tecnológica.

Asimismo, evidencié ausencia del personal calificado en el área tecnológica que acompañe en el aula de clases para el buen manejo y uso del computador, quien, al momento de presentarse fallas con éste pueda habilitar su arreglo.

Por esa razón, surge la inquietud por realizar esta investigación, de carácter interpretativo la cual me permita generar una aproximación teórica que fundamente el uso del Proyecto Educativo Canaima como herramienta para fortalecer la calidad educativa en las escuelas primarias ubicadas en la parroquia Marcelino Briceño, municipio Baralt, estado Zulia interpretando las opiniones de los docentes sobre el tema. De las consideraciones antes mencionadas, surgen las siguientes interrogantes:

¿Cómo perciben los docentes de las escuelas primarias ubicadas en la parroquia Marcelino Briceño, el uso del Proyecto Educativo Canaima?

¿Qué elementos del aprendizaje fortalece el uso de las Canaima en educación primaria? 


\subsection{Propósito de la investigación}

Generar una aproximación teórica que fundamente el uso del Proyecto Educativo Canaima como herramienta para fortalecer la calidad educativa en las escuelas primarias ubicadas en la parroquia Marcelino Briceño, municipio Baralt, estado Zulia.

\section{Contexto Referencial}

\subsection{Proyecto Educativo Canaima}

El Proyecto Educativo Canaima es un instrumento de aprendizaje sumamente enriquecedor, tanto para los estudiantes como los mismos docentes, gracias a que el educando aprenderá de forma más dinámica, contextualizada, pedagógica. A este respecto, Chávez (2010:17) plantea:

El proyecto Canaima, permite la utilización de nuevas herramientas pedagógicas en el proceso de enseñanza y aprendizaje, que utilizadas adecuadamente dará beneficios altamente positivos para la educación venezolana, por esa razón, requiere de personal formado y capacitado en el área de la Informática y teleinformática.

Todo lo anterior, conduce a generar herramientas de apoyo y soluciones educativas tecnológicas con altos niveles de calidad de la educación, a través de la difusión, así como en el uso compartido de la información para consolidar el desarrollo de las potencialidades individuales, colectivas, tanto de los docentes como de los educandos. Cabe considerar que el impacto generado por el Proyecto Educativo Canaima debe orientarse al logro de los fines de la educación venezolana, entre los cuales se plantea la protección de los conocimientos tradicionales, por ello, los objetivos de éste deben corresponder a la consolidación de éstos, asumiéndola no como un procesador de saberes vacío, sin pertenencia local, regional, nacional y tampoco debería generar conflictos a nivel psicológico, social, educativo. Al respecto, Centeno y otros (2011:11) destacan como logros de este proyecto educativo: 
Las Canaima han logrado despertar el interés en los estudiantes sobre todo en la investigación científica, logrando así el mejoramiento de las habilidades creativas, la imaginación, la comunicación, pudiendo acceder a mayor cantidad de información y proporcionando los medios para un mejor desarrollo integral.

Visto de esa forma, el Proyecto Canaima Educativo posibilita nuevos escenarios para fomentar la participación activa de los estudiantes, la familia, comunidad en los procesos pedagógicos acordes con lo previsto en el Currículo Nacional Bolivariano, siendo por tanto, un pilar fundamental en la construcción del nuevo modelo educativo revolucionario. Este programa, y por ende, los procesos implicados en él nacen de los compromisos del Estado Venezolano para dar respuesta a los derechos de los /as venezolanos/as. En ese sentido, tiene sustento jurídico en los artículos 108 y 110 de la Constitución de la República Bolivariana de Venezuela (1999), los cuales establecen que éste es responsable de garantizar servicios de informática, por tanto, los centros educativos deben incorporar el conocimiento, aplicación de las nuevas tecnologías con sus correspondientes innovaciones, por ser instrumento fundamental para el desarrollo económico, social, político del país, así como para la seguridad, soberanía nacional.

Igualmente, el Plan de Desarrollo Económico Social de la Nación (20012007) establece entre los objetivos específicos, disminuir progresivamente las inequidades sociales a través de una educación de calidad para todos/as; así como mejorar, construir sistemas de comunicación e información. Asimismo, el Proyecto Nacional Simón Bolívar, Primer Plan Socialista de la Nación (20072013) establece en la Línea II: la Suprema Felicidad Social, como estrategia y política para profundizar la universalización de la educación bolivariana, incorporando las TIC al proceso educativo.

Por otra parte, la Ley Orgánica de Ciencia Tecnología e Innovación (LOCTI, 2005). Establece en su artículo 1, que se tiene por objeto desarrollar 
los principios orientadores en materia de ciencia, tecnología e innovación con sus aplicaciones. Igualmente, la Ley Orgánica para la Protección de Niños, Niñas y Adolescentes (LOPNA, 2007), expresa en el artículo 73:

El Estado debe fomentar la creación, producción y difusión de diversos materiales informativos, libros, publicaciones, obras artísticas y producciones audiovisuales, radiofónicas y multimedia dirigidas a los niños, niñas y adolescentes, que sean de la más alta calidad, plurales y que promuevan los valores de paz, democracia, libertas, tolerancia, igualdad entre las personas y sexos, así como el respeto a sus padres, representantes o responsables y a su identidad nacional y cultural.

En mi opinión, hoy en día el Proyecto Educativo Canaima es una herramienta tecnológica que permite el desarrollo de habilidades que apoyen el aprendizaje, la productividad personal, así como toma de decisiones en la vida diaria de los educandos. Visto de esa forma, el PEC se propone formar un estudiante que sea un actor social activo, creativo, sujeto y objeto de aprendizaje, quien a través de la participación activa en las interacciones sociales con sus compañeros, así como demás miembros de la comunidad

Finalidades del Proyecto Educativo Canaima (PEC): de acuerdo con González (2012) el PEC presenta las siguientes finalidades: a) Lograr la formación de una ciudadanía con identidad propia, construida sobre la reflexión crítica y el análisis creado de su contexto geohistórico cultural, portadora de valores de justicia, integridad, cooperación, dignidad, bien común, paz, solidaridad. b) Generar una nueva cultura política fundamentada en la participación protagónica que permita a todos/as ejercer potencial creativo para enfrentar los cambios, propiciar las transformaciones de la desde los principios de la corresponsabilidad y la sustentabilidad. c) Redimensionar los recursos para el aprendizaje que venían utilizando los docentes/as e incorporar los medios de comunicación social públicos y privados: televisión, radio, prensa, tecnologías de la información, tanto libres como privadas, entre 
otros, como medios para la formación integral, de calidad de los miembros de la comunidad educativa. d) Lograr que los/as docentes hagan uso creativo y crítico de las portátiles Canaima en el sentido de darle versatilidad a los diferentes contenidos incluidos en ellas atreviéndose a generar nuevos contenidos contextualizados.

Estas finalidades, a juicio de la investigadora, otro modo de concebir al ser humano, la educación, el aprendizaje y sus procesos; siendo necesario para ello hacer esfuerzos, idear, redefinir procesos integradores, formadores de las múltiples dimensiones del ser humano. Lo cual no significa incorporar más contenidos, sino pensar, actuar de manera creativa e intencional e ir entrelazando, integrando los correspondientes a las diferentes áreas.

\subsection{Elementos de aprendizaje que se fortalecen con el uso de las Canaima}

EI PEC busca promover la formación de los/as niños/as venezolanos/as mediante el fortalecimiento de diversos elementos de aprendizaje que están plasmados en el Currículo Nacional Bolivariano, los cuales permiten el desarrollo de capacidades, apropiación y promoción del libre conocimiento. Entre estos elementos encontramos: pensamiento crítico, aprendizaje colaborativo, aprendizaje dialógico.

\subsubsection{Pensamiento crítico}

El pensamiento crítico se propone analizar o evaluar la estructura y consistencia de los razonamientos, particularmente opiniones o afirmaciones que la gente acepta como verdaderas en el contexto de la vida cotidiana. Por tanto, es un proceso mediante el cual se usa el conocimiento, así como la inteligencia para llegar efectivamente, a la posición más razonable sobre un determinado tema. En la educación primaria, el pensamiento crítico provee a los estudiantes habilidades como el razonamiento, resolución de problemas y 
toma de decisiones, las cuales les permiten procesar, reelaborar la información que se recibe proporcionando un basamento para sustentar sus ideas, posibilitando la construcción de conocimientos de manera eficaz.

Puede decirse entonces que es un proceso de búsqueda de conocimiento a través de la aplicación de herramientas de razonamiento, solución de problemas, toma de decisiones, las cuales permiten al estudiante la construcción tanto del conocimiento como de las habilidades mentales necesarias para ello. Al respecto, Facione (2010:32) define el pensamiento crítico como el juicio autorregulado, con propósito, dando como resultado interpretación, análisis, evaluación e influencia, como también la explicación de las consideraciones de evidencia, conceptuales en que se basa el mismo.

\subsubsection{Aprendizaje colaborativo}

Los cambios en las concepciones de aprendizaje, así como en las metodologías propuestas en la educación universitaria recomiendan innovaciones orientadas a hacer la enseñanza más práctica, activa, incorporando nuevas metodologías, estrategias educativas. Estos planteamientos demandan la necesidad de dirigir la atención de los estudiantes hacia actividades prácticas, así como la reflexión sobre su proceso de aprendizaje. En relación al aprendizaje en este nuevo escenario, Huber (2008:42), plantea que los procesos educativos basados en la participación activa del estudiante usando el potencial de las tecnologías se orientan a un aprendizaje colaborativo, pues en los ambientes virtuales, el estudiantado se muestra más participativo incrementando de manera significativa la cantidad y calidad de sus intervenciones.

Desde esa perspectiva, se considera que el educador ejerce funciones de feedback educativo, seguimiento, estructuración de la enseñanza, jugando un papel fundamental en la creación del clima de colaboración, cohesión de 
grupos, distribuyéndose de manera similar entre docente y estudiante, por cuanto responde a modelos de enseñanza participativos, de cooperación.

\subsubsection{Aprendizaje dialógico}

EI PEC tiene como marco teórico primordial el aprendizaje dialógico, el cual se basa en las interacciones y el diálogo como herramientas clave, destacando que para promoverlo no sólo son importantes dichas interacciones entre docentes, estudiantes, sino con toda la diversidad de personas con las cuales se relacionan. Al respecto, Elboj y otros (2009:13) señala que el aprendizaje dialógico parte de la inteligencia cultural no sólo de todos los estudiantes sino también de docentes y demás personas con quienes se relacionan. Visto de esa forma, el aprendizaje dialógico fomenta la solidaridad dentro del aula, pues los educandos se ayudan entre ellos en el proceso de construcción colaborativa de saberes, en un diálogo igualitario entre docentes y estudiantes. Aunado a lo antes expuesto, como investigadora considero pertinente, accionar algunas teorías fundamentales que serán descritas posteriormente y que permitan fomentar el desarrollo de potencialidades, habilidades, destrezas, en pro de la democratización del saber, la independencia y la soberanía tecnológica mediante la puesta en práctica de nuevos paradigmas educativos a partir de los cuales la incorporación de las TIC en los procesos educacionales.

\section{Teorías de aprendizaje que fundamentan el uso del PEC}

En un mundo donde el conocimiento, la ciencia y la tecnología juegan un papel de primer orden, el desarrollo y el fortalecimiento de la educación constituyen un elemento insustituible para el avance social, la generación de riqueza, el fortalecimiento de las identidades culturales, la cohesión social, la lucha contra la pobreza, el hambre, la prevención del cambio climático, la crisis energética, así como para la promoción de una cultura de paz. 
Entre las teorías de aprendizaje que sustentan el uso de los entornos virtuales en educación se encuentran: el constructivismo, la pedagogía social, el conectivismo.

\subsection{Constructivismo}

El Constructivismo, señala De la Torres (2011:49), es un enfoque pedagógico que implica "un proceso de ordenación de componentes para construir un conocimiento", esto indica el involucramiento del ser humano integral, no sólo su intelecto. Ahora bien, de acuerdo con Norman (2012:138), el constructivismo se define como "un conjunto de teorías psicológicas que conciben los procesos cognitivos como construcciones eminentemente activas, resultado de la interacción del sujeto con el ambiente, los demás y consigo mismo". Conforme con lo anterior, el enfoque constructivista, basa los éxitos del aprendizaje en la interacción y la comunicación de los estudiantes, así como en el debate, la crítica argumentativa del grupo para lograr resultados cognitivos, éticos, colectivos, además de soluciones a los problemas reales mediante la interacción teórico-práctica.

\subsection{Pedagogía social}

Comienza hacia fines del siglo XIX. Su campo no es homogéneo ni hegemónico. Podríamos decir que tiene un carácter emergente. Desde ese lugar me planteo la pedagogía social como la disciplina pedagógica desde la se trabaja teórica, prácticamente en las complejas fronteras de exclusión /inclusión social. En relación a esas fronteras, se trata de un trabajo de ampliación en lo pedagógico y también en lo político de las dimensiones de las responsabilidades públicas educativas. Puede decirse que la pedagogía social es un modelo teórico donde se plantea la construcción de saberes en colectivo, mediante el contacto con el entorno, en este caso, el entorno virtual, pues 
considera que de esa manera, se produce el aprendizaje verdaderamente significativo.

\section{Metodología}

La presente investigación se realizará bajo el enfoque cualitativo, bajo el método fenomenológico.

Según Lincoln y Guba (2012:17) "la investigación cualitativa, dispone de métodos y técnicas que van a depender de la naturaleza del objeto a estudiar, los cuales se deben adaptar a la realidad donde se enfatiza la comprensión del fenómeno social desde la intersubjetividad con los otros. La misma requiere su abordaje desde la perspectiva de un método capaz de cumplir con las expectativas de poder interpretarla. Por otra parte, deben cumplir con la condición de ser emergente en la estructuración de su diseño, con cualidad interactiva y participativa.

A lo expuesto por los autores se puede decir que los investigadores cualitativos estudian la realidad en su contexto natural, tal como sucede, intentando interpretar, los fenómenos de acuerdo con los significados que tienen para las personas implicadas.

En ese sentido, la investigación cualitativa dentro del presente estudio tiene como propósito generar una aproximación teórica que fundamente el uso del Proyecto Educativo Canaima como herramienta para fortalecer la calidad educativa en las escuelas primarias ubicadas en la parroquia Marcelino Briceño, municipio Baralt, estado Zulia. Dado que la investigación de naturaleza cualitativa, se afianza en los enunciados de Taylor y Bodgan (2011) quienes explican una caracterización de sobre este tipo de investigación, categorizando el estilo para asumir en la búsqueda de la información. 


\section{Análisis de los Resultados.}

\subsection{Técnicas y procedimiento de análisis de datos}

El desarrollo de la investigación se ubica en el marco de una investigación cualitativa bajo un enfoque hermenéutico fenomenológico, que permitirá la aproximación a la realidad estudiada con la suficiente flexibilidad como para considerar los distintos puntos de vista considerados por cada informante de acuerdo a su experiencia particular. En tal sentido, se conciben tres fases principales para el desarrollo de la investigación:

- La teorética, que implica la revisión de las diferentes fuentes bibliográficas a la luz de las cuales se construyeron el referencial teórico del estudio, así como sus fundamentos ontológicos, epistemológicos y metodológicos.

- La fase empírica implica la aplicación de la entrevista y la discusión de su transcripción para ampliar la perspectiva de los informantes en torno a la realidad del uso del Proyecto Educativo Canaima como herramienta para fortalecer la calidad educativa en las escuelas primarias.

- La fase de análisis de la información que se hará por medio del análisis del contenido de las entrevistas para hacer inferencias sobre los contenidos simbólicos y significativos que se encuentran en ellas para luego organizarlos en estructuras sistemáticas creando categorías a partir de los aspectos que emergerán de la información aportada por los informantes.

\subsection{Procedimiento utilizado para realizar las entrevistas}

Bajo esta mirada, la entrevista en profundidad a propósito de la información requerida a la presente investigación, tiene cabida dado a ese intercambio permanente de diálogos donde el entrevistado forma parte esencial del proceso de construcción de conocimientos desde su experiencia de vida, su lado profundo de pensamiento en conexión con la subjetividad, permitiendo acceder a su ser. Precisamente, a través del manejo de la 
entrevista en profundidad, procedí a la grabación de voz, con ayuda de una grabadora, en varios encuentros conversacionales con los participantes, destacándose el hecho de que todas las entrevistas realizadas donde a diario laboran los docentes seleccionados, obedeciendo igualmente a la disponibilidad de los mismos: patio central de las escuelas, en las bancas que allí se encuentran, por petición de los mismos, quienes comentaron, desde sus propios criterios y fuera de la grabación, sentirse cómodos en el sitio, e identificados en su espacio habitual.

Dentro de ese contexto, los docentes adoptan posturas que les facilitaban el intercambio de palabras al momento de la entrevista. Previamente, pensé en utilizar la biblioteca para el confort de los docentes, sin embargo, de preferencia quisieron realizar las entrevistas al aire libre. Sin embargo, las ideas previas que promoverán un proceso de interacción con los estudiantes, darán paso al discurso de los mismos, a partir de preguntas de: ¿Qué opinión tienes del Proyecto Educativo Canaima?, ¿Crees que ese proyecto está dando resultado?, ¿Cuáles teorías de aprendizaje asocias al uso de la Canaima?, ¿En tu opinión que elementos del aprendizaje fomentan el uso de la Canaima?, ¿Posees conocimientos sobre software libres? donde surgen vivencias, experiencias e igualmente se destacan las motivaciones.

\subsection{Proceso de transcripción e interpretación de las entrevistas realizadas.}

Según los propósitos de la investigación y mi interés particular como investigadora, realicé el primer contacto con los estudiantes en sus propias aulas de clase, donde les expliqué brevemente los fines para los cuales se requería de entrevistas partiendo además, de la consideración inicial de las ideas que surgieron de mi propia experiencia, dadas las razones voluntariamente accedieron a formar parte del proceso en estudio, las cuales darán paso a un proceso de diálogo donde es considerada la entrevista en 
profundidad, por las características anteriormente descritas. Por lo tanto el objetivo central del método de análisis utilizado es la construcción de una teoría y, de acuerdo con los autores mencionados (ob.cit.:159), se entiende "que el analista reduce los datos de muchos casos a conceptos y los convierte en conjuntos de afirmaciones de relación que pueden usarse para explicar, en un sentido general, lo que ocurre". Para ello se elegirá, en forma excluyente, la unidad de información apoyada por el Verbatum de los entrevistados que, con claridad, delimitaba los aspectos analizados, se reorganizarán las categorías y subcategorías, las cuales se definirán a partir de las anotaciones de las distintas ideas surgidas durante el análisis de las narrativas, las cuales darán cuenta de las vivencias en relación al uso del Proyecto Educativo Canaima como herramienta para fortalecer la calidad educativa en las escuelas primarias ubicadas en la parroquia Marcelino Briceño, municipio Baralt, estado Zulia.

\section{Conclusiones}

Como investigadora de este relevante tópico, puedo generar las siguientes conclusiones:

Según los propósitos de la investigación y mi interés particular como investigadora, realice el primer contacto con los estudiantes en sus propias aulas de clase, donde les expliqué brevemente los fines para los cuales se requería de entrevistas partiendo además, de la consideración inicial de las ideas que surgieron de mi propia experiencia, dadas las razones voluntariamente accedieron a formar parte del proceso en estudio, las cuales darán paso a un proceso de diálogo donde es considerada la entrevista en profundidad, por las características anteriormente descritas.

En ese sentido, una vez grabadas las entrevistas serán transcritas a través de un formato computarizado en cuadros que se dividirán en: número de línea, representando la línea discursiva del momento, unidades discursivas 
en referencia al contenido del discurso del entrevistado, y categorías donde se refleja mi interpretación al momento de su análisis; todo esto en estilo de Horizontalidad, bajo el modelo de Moustakas (2012) desde sus constructos de la fenomenología, los cuales serán descritos más adelante; es de este modo como quedaron conformados los cuadros de representación visual de las entrevistas. Asimismo, para analizar los datos se utilicé el método de las comparaciones constantes propuestas por Strauss \& Corbin (2010:25) el cual se caracteriza por la generación de teoría que emerge de los datos, definido como un "conjunto de categorías bien construidas, por ejemplo, temas y conceptos, interrelacionadas de manera sistemática por medio de oraciones que indican relaciones, para formar un marco teórico que explica algún fenómeno social, psicológico, educativo, de enfermería o de otra clase".

La teoría permite establece una nueva red de relaciones entre las partes constituyentes, construidas a partir de la organización de los hechos observados, analizados, representados conceptualmente, constituyendo el aporte específico de la investigación. Durante el proceso de análisis, se entrecruzarán los datos codificándolos de dos formas: abierta y axial, analizando e interpretando la información cualitativa a partir de las entrevistas en profundidad realizados durante el estudio. La codificación abierta, en opinión de los autores antes mencionados, constituye uno de los pasos necesarios para la construcción de la teoría, hacer las conceptualizaciones, definir las categorías y desarrollarlas en los términos de sus propiedades y dimensiones, para luego relacionarlas por medio de hipótesis o relaciones que establecen las dimensiones.

Entretanto, la codificación axial permite depurar y diferenciar las categorías derivadas de la codificación abierta. De todas las categorías que se originen se toman las que son más ricas y prometedoras en información para una elaboración adicional. Estas categorías axiales se enriquecen en la medida que tengan más número de testimonios o pasajes posibles en el texto, 
para perfeccionarlas se utilizan las preguntas y comparaciones. Por último, se desarrollan las comparaciones entre éstas y otras categorías estableciéndose la relación a su vez entre categorías y subcategorías.

El método de comparaciones constantes se utilizó a través de cuatro fases: comparaciones de fragmentos de entrevistas, integración de categorías y sus propiedades, delimitación construcción, escritura de la teoría. El análisis se realizó con base en la narrativa de los sujetos que, de acuerdo con Bruner (2011:53) hacen referencia a una forma de relato para describir acontecimientos de la vida humana: "el relato se imbrica con la vida de la cultura, e inclusive se vuelve parte integrante de ella" y, el autor añade, la fecundidad del lenguaje permite narrar distintas versiones fundamentales para las interacciones sociales. Para la aplicación del método de análisis se realicé en primer lugar, de acuerdo con Strauss \& Corbin (2010:111) la codificación abierta para lo cual se transcribieron las entrevistas, luego se procederá a editar las transcripciones, releerlas y destacar los segmentos del texto compartidos en distintos párrafos con el fin de identificar las teorías emergentes. Durante ésta "los acontecimientos, sucesos, objetos y acciones que se consideran conceptualmente similares en su naturaleza o relacionados en el significado se agrupan bajo conceptos más abstractos denominados categorías".

Durante el proceso de codificación se examinaron y compararon los datos para buscar semejanzas y diferencias. La indagación inicial de los datos brutos permitirá la construcción de unas categorías, así como subcategorías iniciales. En segundo lugar, se procederá a organizar la codificación axial, la cual según Strauss \& Corbin (2010:135), tiene como propósito "comenzar el proceso de reagrupar los datos que se fracturaron durante la codificación abierta".

Para ello se elegirá, en forma excluyente, la unidad de información apoyada por el Verbatum de los entrevistados que, con claridad, delimitaba los 
aspectos analizados, se reorganizarán las categorías y subcategorías, las cuales se definirán a partir de las anotaciones de las distintas ideas surgidas durante el análisis de las narrativas, las cuales darán cuenta de las vivencias en relación al uso del Proyecto Educativo Canaima como herramienta para fortalecer la calidad educativa en las escuelas primarias ubicadas en la parroquia Marcelino Briceño, municipio Baralt, estado Zulia.

\section{Referencias}

Bruner, J. (2011). “La fábrica de historias. Derecho, literatura, vida”. Fondo de Cultura Económica. Buenos Aires.

Constitución de la República Bolivariana de Venezuela (1999). Gaceta oficial № 36.830. Caracas.

González, M. (2012). "La Canaima educativa una herramienta tecnológica de enseñanza en la educación primaria bolivariana". Campus Virtual UFT. Barquisimeto.

Ley Orgánica para la Protección de Niños, Niñas y Adolescentes (2007). Gaceta Oficial № 5.859. Caracas.

Lincoln, Y. y Guba, E. (2012). "Paradigmas competentes en investigación cualitativa". Editorial Sage. Londres.

Moustakas, C. (2012). "Métodos de investigación fenomenológica". Editorial Eunsa. Madrid.

Norman, A. (2012). "Aprender a aprender". Editorial Paidós. Barcelona, España.

Proyecto Nacional Simón Bolívar (2007-2013). Primer Plan Socialista de la Nación. Caracas.

Taylor, S. y Bogdan, R. (2011). "Introducción a los métodos cualitativos de investigación”. Editorial Paidós. Barcelona.

Torres, R. (2011). “Constructivismo y cognición”. Editorial Limusa. México. 


\section{Eglis Coromoto Quintero Gómez}

e-mail: eglis2311@hotmail.com

Nació en Pueblo Nuevo Estado Zulia, el 23/11/1986.

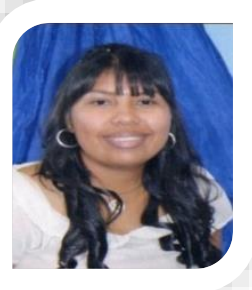

Estudiante de la Universidad Nacional Experimental "RAFAEL MARÍA BARALT" en el DOCTORADO EN EDUCACIÓN. Estudios de postgrado: UNIVERSIDAD NACIONAL EXPERIMENTAL RAFAEL MARÍA BARALT (UNERMB) título obtenido: MAGISTER SCIENSIARIUM EN GERENCIA DE RECURSOS HUMANOS. Estudio de Pregrado: Universidad "ALONSO DE OJEDA" Título Obtenido: LICENCIADA EN EDUCACIÓN PREESCOLAR. Actualmente se desempeña como docente de Aula en el CEIB Don "JOSÉ MARIANO NÚÑEZ DE CÁCERES ubicado en San Rafael de El Tigre, Municipio Baralt Estado Zulia desde 2007 hasta la actualidad.

El contenido de este manuscrito se difunde bajo una Licencia de Creative Commons ReconocimientoNoComercial-Compartirlgual 4.0 Internacional 